\title{
BANACH ALGEBRAS WITH NON-HAUSDORFF STRUCTURE SPACES
}

\author{
BERTRAM YOOD
}

(Communicated by Palle E. T. Jorgensen)

\begin{abstract}
An example due to G. W. Mackey is an instance of a class of Banach algebras whose spaces of primitive ideas are not Hausdorff spaces.
\end{abstract}

\section{INTRODUCTION}

Let $\mathfrak{P}(A)$ be the structure space (space of primitive ideals) of a Banach algebra $A$. Concerning $\mathfrak{P}(A)$ a standard reference on Banach algebras [7, p. 135] stated in 1973 is: "The topology of the structure space has not so far been very effectively related to the algebraic properties of general Banach algebras, ... ". Partly in response to this statement we related in [6] $\mathfrak{P}(A)$ being a discrete space and $\mathfrak{P}(A)$ having a dense set of isolated points with the algebraic nature of $A$.

From the beginning of Banach algebra theory there has been at hand a prominent example of a Banach algebra whose structure space (space of primitive ideals) is not a Hausdorff space. This example is the disc algebra (see $[1 ; 5, \mathrm{p}$. 303]). That structure space (there the space of maximal ideals) is even antiHausdorff in the sense that the intersection of any two nonempty open subsets is nonempty.

We show first that a Banach algebra $A$ has a structure space which is antiHausdorff if and only if its radical is a prime ideal in $A$. Such Banach algebras also play a vital role in the development which we now describe.

Let $W$ be a closed subalgebra of a Banach algebra $A$. We study $B=$ $c(A, W)$ which consists of all sequences $\left\{x_{n}\right\}$ where each $x_{n} \in A$ and $\lim x_{n} \in$ $W$. This is a Banach algebra under the usual rules for combining sequences where $\left\{x_{n}\right\}\left\{y_{n}\right\}=\left\{x_{n} y_{n}\right\}$, and the norm is given by $\left\|\left\{x_{n}\right\}\right\|=\sup \left(\left\|x_{n}\right\|\right)$.

For each primitive ideal $Q$ of $W$ the set of all sequences $\left\{x_{n}\right\}$ in $B$ where $\lim x_{n} \in Q$ is a primitive ideal of $B$ (see $\S 3$ ). Such a primitive ideal we call a limit primitive ideal of $B$. Our interest in these ideals was aroused by an example due to G. W. Mackey described in [5, p. 82]. There $A$ is the set of all two-by-two matrices over the complex field and $W$ is the set of all its diagonal matrices. Then $B$ has two distinct limit primitive ideals $K_{1}$ and $K_{2}$ which are an anti-Hausdorff pair in the sense that each open subset in the structure space containing $K_{1}$ intersects nonvacuously each open subset containing $K_{2}$.

Received by the editors November 18, 1992 and, in revised form, May 3, 1993.

1991 Mathematics Subject Classification. Primary 46H10. 
We show that if either $A$ or $W$ has an anti-Hausdorff structure space then any two limit primitive ideals of $B$ form an anti-Hausdorff pair. This allows us to have a rather natural commutative example where we have such pairs. Let $A=W$ be the disc algebra, and let $z_{1} \neq z_{2}$ be two points in the disc. Let $P_{k}$ be the set of all sequences $\left\{f_{n}\right\}$ in $B$, such that $\left(\lim f_{n}\right)\left(z_{k}\right)=0, k=1,2$. Then $P_{1}$ and $P_{2}$ form an anti-Hausdorff pair in $\mathfrak{P}(B)$.

On the other hand, we show that if $W$ is a two-sided ideal in $A$ which does not have an anti-Hausdorff structure space then there is a pair of limit primitive ideals of $B$ which is not an anti-Hausdorff pair.

\section{ANTI-HAUSDORFF STRUCTURE SPACES}

Let $R$ be a ring and $J$ be the radical of $R$. Let $\mathfrak{P}(R)$ denote the structure space of $R$ (the space of primitive ideals of $R$ ) topologized as in [3, Chapter IX]. For a subset $\mathfrak{S}$ of $\mathfrak{P}(R)$ the closure of $\mathfrak{S}$ is the set of $Q \in \mathfrak{P}(R)$ for which $Q \supset \bigcap\{P \in \mathfrak{P}(R): P \in \mathfrak{S}\}$. Then a set in $\mathfrak{P}(R)$ of the form $\{P \in \mathfrak{P}(R): x \notin$ $P\}$, for an element $x \in R$, is clearly an open subset of $\mathfrak{P}(R)$. These open sets, as $x$ ranges over $R$, form a basis for the open subsets of $\mathfrak{P}(R)$ (see [2, p. 18]). A basic open set $\{P \in \mathfrak{P}(R): x \notin P\}$ is nonempty if and only if $x \notin J$.

Lemma 2.1. For a prime ideal $P$ in a ring $R$ we have $P=\{x \in R: R x R \subset P\}$. Proof. We may suppose that $P$ is a proper ideal in $R$. Let $K$ be the set of $x \in R$ for which $R x R \subset P$. Clearly $K \supset P$. Suppose that $w \in R$ and $w \notin P$. As $P$ is a prime ideal, there exists $y \in R$ such that $w y w \notin P$. Hence $w R \not \subset P$. As $P$ is a proper ideal, we have $R \not \subset P$. Then by [4, Theorem 4.3] we cannot have $R w R \subset P$. Therefore, $K \subset P$.

Lemma 2.2. The radical $J$ is a prime ideal in $R$ if and only if for every $a$, $b \in R$ where $a \notin J, b \notin J$ there is some $P \in \mathfrak{P}(R)$ with $a \notin P$ and $b \notin P$.

Proof. Let $J$ be a prime ideal in $R$. Suppose that for each $P \in \mathfrak{P}(R)$ either $a \in P$ or $b \in P$. Then $a R b \subset P$ for each $P \in \mathfrak{P}(R)$ so that $a R b \subset J$. Then either $a \in J$ or $b \in J$.

Assume that for each $a \notin J, b \notin J$ we have some $P \in \mathfrak{P}(R)$ where $a \notin P$ and $b \notin P$. If we take $v R w \subset J$ for $v, w \in R$ we must show that either $v \in J$ or $w \in J$. Now, for each $P \in \mathfrak{P}(R)$, we have $R v R R w R \subset P$. Then either $R v R \subset P$ or $R w R \subset P$. By Lemma 2.1 we see that either $v \in P$ or $w \in P$. Then by our assumption we see that either $v \in J$ or $w \in J$.

Theorem 2.3. $\mathfrak{P}(R)$ is an anti-Hausdorff space if and only if $J$ is a prime ideal in $R$.

Proof. Let $\left\{P \in \mathfrak{P}(R): a_{k} \notin P\right\}, k=1,2$, be two nonempty basic open subsets of $\mathfrak{P}(R)$ so that each $a_{k} \notin J$. Their interaction is nonempty if and only if there is some $Q \in \mathfrak{P}(R)$ where $a_{k} \notin Q, k=1,2$. By Lemma 2.2 $\mathfrak{P}(R)$ is then an anti-Hausdorff space if and only if $J$ is a prime ideal in $R$.

Corollary 2.4. Let $W$ be a two-sided ideal in $R$. If $\mathfrak{P}(R)$ is an anti-Hausdorff space, then so is $\mathfrak{P}(W)$.

Proof. Let $a$ and $b$ be in $W$, but neither in the radical $W \cap J$ of $W$. Then, by Lemma 2.2 and Theorem 2.3 , there is some $P \in \mathfrak{P}(R)$ where $a \notin P$ and $b \notin P$. However, by [5, Theorem 2.6.6], $P \cap W$ is a primitive ideal of $W$ 
not containing either $a$ or $b$. Hence, $\mathfrak{P}(W)$ is an anti-Hausdorff space by Theorem 2.3.

\section{ON $\mathfrak{P}(B)$ FOR $B=c(A, W)$}

We employ the notation of $\S 1$. First we determine the nature of the primitive ideals of $B$. For each positive integer $m$ let $K_{m}$ be the set of all sequences $\left\{x_{j}\right\}$ in $B$ where $x_{j}=0$ for $j \neq m$. Then $K_{m}$ is a closed two-sided ideal of $B$ identifiable with $A$. Let $\Gamma \in \mathfrak{P}(B)$ where $\Gamma \not \supset K_{m}$. Then $\Gamma \cap K_{m}$ is a primitive ideal of $K_{m}$ by [3, Proposition 2, p. 206]. Now let $Z_{m}$ be the set of all $\left\{x_{j}\right\} \in B$ where $x_{m}=0$. This is a two-sided ideal of $B$. As $K_{m} Z_{m}=(0)$ and $K_{m} \not \subset \Gamma$ then $Z_{m} \subset \Gamma$. Hence, there is $P \in \mathfrak{P}(A)$ so that $\Gamma$ is the set of all $\left\{x_{j}\right\} \in B$ where $x_{m} \in P$. We call this set $P(m)$. Conversely, let $P_{0} \in \mathfrak{P}(A)$. By [3, Proposition 2, p. 206] there is $\Gamma_{0} \in \mathfrak{P}(B)$ where $\Gamma_{0} \cap K_{m}$ is the set of $\left\{x_{j}\right\}$ where $x_{m} \in P_{0}$ and $x_{j}=0$ for $j \neq m$. Reasoning as above we see $\Gamma_{0} \supset Z_{m}$ so that $\Gamma_{0}=P_{0}(m)$.

Suppose now $\Gamma \in \mathfrak{P}(B)$ and $\Gamma$ contains every $K_{m}$. Then $\Gamma$ contains every $\left\{x_{j}\right\}$ where $x_{j}=0$ for all but a finite number of indices. Now $\Gamma$ is closed so that $\Gamma \supset L$ where $L$ is the set of $\left\{x_{j}\right\}$ with $\lim x_{j}=0$. Of course, $B / L$ is isomorphic to $W$. By [3, Proposition 1, p. 205] $\Gamma / L$ is isomorphic to a primitive ideal of $W$. Thus, there is a primitive ideal $P$ of $W$ so that $\Gamma$ is the set of all $\left\{x_{j}\right\} \in B$ where $\lim x_{j} \in P$. Conversely any such set is a primitive ideal of $B$. We refer to the primitive ideals just described as limit primitive ideals of $B$.

Theorem 3.1. Suppose that either $\mathfrak{P}(A)$ or $\mathfrak{P}(W)$ is an anti-Hausdorff space. Let $\mathfrak{G}_{1}$ and $\mathfrak{G}_{2}$ be open sets in $\mathfrak{P}(B)$ each containing a limit primitive ideal. Then $\mathfrak{G}_{1} \cap \mathfrak{G}_{2}$ is nonempty.

Proof. Let $\Gamma_{k}$ be a limit primitive ideal of $B, k=1,2$. There exist $Q_{k} \in$ $\mathfrak{P}(W), k=1,2$, so that

$$
\Gamma_{k}=\left\{\left\{x_{k}\right\} \in B: \lim x_{k} \in Q_{k}\right\}, \quad k=1,2 .
$$

Let $\mathscr{V}_{k}$ be a basic open neighborhood of $\Gamma_{k}$ in $\mathfrak{P}(B), k=1,2$. Then there exists a sequence $\left\{y_{k}, j\right\}, j=1,2, \ldots, k=1,2$, where

$$
\mathscr{V}_{k}=\left\{\Gamma \in \mathfrak{P}(B):\left\{y_{k, j}\right\} \notin \Gamma\right\}, \quad k=1,2 .
$$

As $\Gamma_{k} \in \mathscr{V}_{k}$ we see that $\lim _{j} y_{k, j} \notin Q_{k}, k=1,2$.

Suppose first the $\mathfrak{P}(A)$ is an anti-Hausdorff space. We must show that $\mathscr{V}_{1} \cap \mathscr{V}_{2}$ is nonempty. Consider the case where, for some positive integer $m, y_{1, m} \notin J$ and $y_{2}, m \notin J$. By Lemma 2.2 and Theorem 2.3 there is a primitive ideal $P_{0}$ of $A$ so that $y_{1, m} \notin P_{0}$ and $y_{2, m} \notin P_{0}$. But then $P_{0}(m)$ lies in $\mathscr{V}_{1} \cap \mathscr{V}_{2}$.

Therefore, $\mathscr{V}_{1} \cap \mathscr{V}_{2}$ is not empty if we rule out the possibility that, for each positive integer $n$, either $y_{1, n} \in J$ or $y_{2, n} \in J$. In that case either $y_{1, n} \in J$ for infinitely many integers $n$ or $y_{2, n} \in J$ does so. Say $y_{1, n} \in J$ for infinitely many $n$. Then $\lim y_{1, n} \in J$. But then $\lim y_{1, n} \in J \cap W$, which is in the radical of $W$; whence $\lim y_{1, n} \in Q_{1}$, contrary to our set-up.

Suppose next that $\mathfrak{P}(W)$ is an anti-Hausdorff space. Now $\lim y_{k, n} \notin Q_{k}$, $k=1,2$, so that by Lemma 2.2 and Theorem 2.3 there is some $Q_{3} \in \mathfrak{P}(W)$ 
where $\lim y_{k, n} \notin Q_{3}, k=1,2$. But then $\mathscr{V}_{1} \cap \mathscr{V}_{2}$ must contain that primitive ideal of $B$ made up of all $\left\{x_{j}\right\} \in B$ where $\lim x_{j} \in Q_{3}$.

In the example of $[5, \mathrm{p} .82] \mathfrak{P}(A)$ is an anti-Hausdorff space and $\mathfrak{P}(W)$ is not. A case for Theorem 3.1 where $\mathfrak{P}(A)$ is not an anti-Hausdorff space and $\mathfrak{P}(W)$ is one comes with the choice for $A$ as the set of continuous functions on the unit disc in the complex plane and $W$ as the disc algebra.

Theorem 3.2. Suppose that $W$ is a closed two-sided ideal of $A$. There exist two limit primitive ideals of $B=c(A, W)$ which do not form an anti-Hausdorff pair if and only if $\mathfrak{P}(W)$ is not an anti-Hausdorff space.

Proof. Note that by Corollary $2.4, \mathfrak{P}(W)$ must be an anti-Hausdorff space if $\mathfrak{P}(A)$ is. By Theorem 3.1 it is enough to show the "if" statement. Suppose that $\mathfrak{P}(W)$ is not an anti-Hausdorff space. The radical of $W$ is $J \cap W$. From Lemma 2.2 and Theorem 2.2 we see that there exist elements $a$ and $b$ in $W$ neither in $J \cap W$ so that every $Q \in \mathfrak{P}(W)$ contains at least one of $a$ and $b$.

There must be some $Q_{1} \in \mathfrak{P}(W)$ where $a \notin Q_{1}$ as $a$ is not in the radical of $W$. Then $b \in Q_{1}$. Likewise there is $Q_{2} \in \mathfrak{P}(W)$ where $b \notin Q_{2}$ and $a \in Q_{2}$.

Consider two elements $\left\{a_{j}\right\}$ and $\left\{b_{j}\right\}$ in $B$ where $a_{j}=a$ and $b_{j}=b$ for each positive integer $j$. Take the sets in $\mathfrak{P}(B)$ :

$$
\mathscr{V}_{1}=\left\{\Gamma \in \mathfrak{P}(B):\left\{a_{j}\right\} \notin \Gamma\right\}, \quad \mathscr{V}_{2}=\left\{\Gamma \in \mathfrak{P}(B):\left\{b_{j}\right\} \notin \Gamma\right\} .
$$

$\mathscr{V}_{1}$ is a neighborhood of the limit primitive ideal of all $\left\{v_{j}\right\} \in B$ where $\lim v_{j} \in$ $Q_{1}$, and $\mathscr{V}_{2}$ is a neighborhood of the limit primitive ideal of all $\left\{w_{j}\right\} \in B$ where $\lim w_{j} \in Q_{2}$. We show that $\mathscr{V}_{1} \cap \mathscr{V}_{2}$ is empty.

We first examine $\Gamma \in \mathfrak{P}(B)$ which is not a limit primitive ideal. There is some $P \in \mathfrak{P}(A)$ and a positive integer $m$ so that $\Gamma=P(m)$. If $P \supset W$, then we see that $\left\{a_{j}\right\} \in \Gamma$ and $\left\{b_{j}\right\} \in \Gamma$. If $P \not \supset W$, then, as $P \cap W \in \mathfrak{P}(W)$, at least one of $a$ and $b$ is $P$. Hence, either $\left\{a_{j}\right\} \in \Gamma$ or $\left\{b_{j}\right\} \in \Gamma$. In either case $\Gamma$ fails to be in at least one of $\mathscr{V}_{1}$ and $\mathscr{V}_{2}$.

Next suppose $\Gamma$ is a limit primitive ideal of $B$. There is $Q \in \mathfrak{P}(W)$ so that $\Gamma=\left\{\left\{x_{j}\right\}: \lim x_{j} \in Q\right\}$. But $Q$ must contain at least one of $a$ and $b$ so that again $\Gamma$ fails to be in one of $\mathscr{V}_{1}$ or $\mathscr{V}_{2}$.

\section{ON ISOLATED POINTS IN $\mathfrak{P}(B)$}

In the special case of the Mackey example [5, p. 82] the isolated points of $\mathfrak{P}(B)$ are determined. We examine the isolated points of $\mathfrak{P}(B)$. Let $\mathfrak{I}(A)$ be the set of isolated points of $A$ and $\mathfrak{I}(B)$ those of $B$. The Mackey example has the property that $\mathfrak{I}(B)$ is dense in $\mathfrak{P}(B)$ and $\mathfrak{I}(B) \neq \mathfrak{P}(B)$. We show that if $\mathfrak{I}(A)$ is dense in $\mathfrak{P}(A)$ and $W$ is not a radical algebra, then $\mathfrak{I}(B)$ is dense in $\mathfrak{P}(B)$ and $\mathfrak{I}(B) \neq \mathfrak{P}(B)$.

Lemma 4.1. If $P \in \mathfrak{I}(A)$ then $P(m) \in \mathfrak{I}(B)$. If $P$ is in the closure of $\mathfrak{I}(A)$ then $P(m)$ is in the closure of $\mathfrak{I}(B)$.

Proof. Suppose that $P \in \mathfrak{I}(A)$. Select $w \in A$ so that $\{Q \in \mathfrak{P}(A): w \notin Q\}$ is an open set in $\mathfrak{P}(A)$ containing just $P$. Thus $w \in Q$ for all $Q \in \mathfrak{P}(A)$, $Q \neq P$. We consider the sequence $\left\{x_{j}\right\}$ where $x_{m}=w$ and $x_{j}=0$ for $j \neq m$, and set $\mathscr{V}=\left\{\Gamma \in \mathfrak{P}(B):\left\{x_{j}\right\} \notin \Gamma\right\}$. This is a neighborhood of $P(m)$. As $\left\{x_{j}\right\}$ lies in every $Q(m), Q \neq P$ in $\mathfrak{P}(A)$, in every $P(j), j \neq m$, and in every limit primitive ideal of $B$, we see that $P(m) \in \mathfrak{I}(B)$. 
Now suppose that $P$ is in the closure of $\mathfrak{I}(A)$. Let $\mathscr{V}=\{\Gamma \in \mathfrak{P}(B)$ : $\left.\left\{y_{j}\right\} \notin \Gamma\right\}$ be a neighborhood of $P(m)$ so that $y_{m} \notin P$. Then $\{Q \in \mathfrak{P}(A)$ : $\left.y_{m} \notin Q\right\}$ contains some $Q_{0} \in \mathfrak{I}(A)$. Then $\left\{y_{j}\right\} \notin Q_{0}(m)$ so that $Q_{0}(m) \in \mathscr{V}$. By the above we have $Q_{0}(m) \in \mathfrak{I}(B)$.

Lemma 4.2. The radical $K$ of $B$ is the set of all $\left\{x_{n}\right\}$ in $B$ where every $x_{n} \in J$. Proof. Let $\left\{x_{n}\right\} \in K$. As $\left\{x_{n}\right\} \in \bigcap\{P(m): P \in \mathfrak{P}(A)\}$ we see that $x_{m} \in J$ for each positive integer $m$. Suppose every $x_{n} \in J$ and $\left\{x_{n}\right\} \in B$. Clearly $\left\{x_{n}\right\}$ lies in every $P(m), P \in \mathfrak{P}(A)$. Now $W \cap J$ is an ideal in $W$ all of whose elements have spectral radius zero. Thus $W \cap J$ is contained in the radical of $W$. Hence, $\lim x_{n}$ lies in that radical so that $\left\{x_{n}\right\}$ lies in every limit primitive ideal of $B$. Thus $\left\{x_{n}\right\} \in K$.

In particular, $B$ is semisimple if $A$ is semisimple.

Theorem 4.3. If $\mathfrak{I}(A)$ is dense in $\mathfrak{P}(A)$, then $\mathfrak{I}(B)$ is dense in $\mathfrak{P}(B)$. No limit primitive ideal of $B$ is in $\mathfrak{I}(B)$.

Proof. Suppose that $\mathfrak{I}(A)$ is dense in $\mathfrak{P}(A)$. For each positive integer $m$ set

$$
T_{m}=\bigcap\{P(m): P \in \mathfrak{I}(A)\} .
$$

Then $T_{m}$ is the set of all $\left\{x_{n}\right\} \in B$ where $x_{m} \in J$. By Lemma 4.1 the intersection of all the $\Gamma \in \mathfrak{I}(B)$ is contained in the intersection of all the sets $T_{m}$. But that is, by Lemma 4.2, the radical of $B$. Hence, $\mathfrak{I}(B)$ is dense in $\mathfrak{P}(B)$.

Let $\Gamma_{0}$ be a limit primitive ideal of $B$ where $\Gamma_{0}$ is the set of all $\left\{x_{n}\right\}$ in $B$ with $\lim x_{n} \in Q$ for some $Q \in \mathfrak{P}(W)$. Let $\mathscr{V}=\left\{\Gamma \in \mathfrak{P}(B):\left\{y_{n}\right\} \notin \Gamma\right\}$ be an open neighborhood of $\Gamma_{0}$ in $\mathfrak{P}(B)$. As $\Gamma_{0} \in \mathscr{V}$, we have $\lim y_{n} \notin Q$. It cannot happen that every $y_{n} \in J$, for otherwise $\lim y_{n} \in W \cap J$ which is contained in the radical of $W$. There is then a positive integer $m$ where $y_{m} \notin J$. For some $P \in \mathfrak{P}(A)$ we have $y_{m} \notin P$. Consequently $P(m) \in \mathscr{V}$ so that $\Gamma_{0}$ cannot be in $\mathfrak{I}(B)$.

\section{REFERENCES}

1. I. M. Gelfand and G. E. Shilov, Über verschiedene Methoden der Einführung der Topologie in die Menge der maximalen Ideale eines normierten Ringen, Mat. Sb. 9 (1941), 25-39.

2. C. W. Kohls, The space of prime ideals of a ring, Fund. Math. 45 (1957), 17-27.

3. N. Jacobson, Structure of rings, Amer. Math. Soc. Colloq. Publ., vol. 37, Amer. Math. Soc., Providence, RI, 1956.

4. N. H. McCoy, The theory of rings, Macmillan, New York, 1964.

5. C. E. Rickart, General theory of Banach algebras, Princeton Univ. Press, Princeton, NJ, 1960.

6. B. Yood, Structure spaces of rings and Banach algebras, Michigan Math. J. 31 (1984), 181-189.

7. F. F. Bonsall and J. Duncan, Complete normed algebras, Springer, New York, 1973.

Department of Mathematics, Pennsylvania State University, University Park, PennSYLVANIA 16802 